This article may be downloaded for personal use only.

Any other use requires prior permission of the author and AIP

Publishing.

This article appeared in:

J. Chem. Phys. 148, 034107 (2018)

and may be found at https://doi.org/10.1063/1.5000783 


\title{
Converging many-body correlation energies by means of sequence extrapolation
}

\author{
J. Segarra-Mart' ', ${ }^{1}$ M. Garavelli, ${ }^{2}$ and F. Aquilante ${ }^{3, a), b)}$ \\ ${ }^{1}$ Laboratoire de Chimie UMR 5182, ENS de Lyon, 46 Allee' d'Italie, 69364 Lyon Cedex 07, France \\ ${ }^{2}$ Dipartimento di Chimica Industriale "Toso Montanari," Universita` di Bologna, Viale del Risorgimento 4, 40136 \\ Bologna, Italy \\ ${ }^{3}$ Dipartimento di Chimica "Giacomo Ciamician," Universita` di Bologna, Via F. Selmi 2, 40126 Bologna, Italy
}

\begin{abstract}
We present an extrapolation scheme for the correlation energy in many-body theory that requires only a relatively small fraction of the orbitals spanning the virtual space but recovers nearly the whole dynamic correlation energy, independently of the single- or multi-reference nature of the problem. Applications to both ground-state chemistry and photochemistry are discussed that clearly show how such an extrapolation scheme can be used to overcome the scaling walls in $a b$ initio quantum chemistry arising from the sheer number of molecular orbitals needed for very accurate calculations.
\end{abstract}

\section{INTRODUCTION}

According to a definition attributed to Lowdin," correlation energy is the name given to the difference between the exact non-relativistic energy of an $N$-electron system and its Hartree-Fock (HF) limit. It is customary to identify two components of the correlation energy (dynamic and static) very different in nature and that require specific techniques of approximation. In particular, with a suitable choice of the mean-field approximation, any correlation problem can be brought to a form where only dynamic correlation is miss-ing, a rationale used in multiconfigurational approaches such as the complete active space self-consistent field (CASSCF) method. ${ }^{1-3}$ Static correlation manifests itself in a significant deviation of the correlated electron density from the one given by the HF approximation. This deviation from the HF approximation is often localised in a small portion of the molecule, such as, for instance, the metal core in transition-metal complexes, which enables the successful recovery of most of the static correlation effects while treating only a fraction of the molecular frame, i.e., the metal and closest and directly coordinated ligands. This also applies to organic systems, where the static correlation treatment is reserved for doubly and triply bonded instances, which display localised and pronounced deviations from the HF approximation. In such typical cases, an accurate estimate of the correlation energy thus relies on recovering as accurately as possible the remaining correlation, the dynamic component, which expands throughout the whole molecular system.

Whereas static correlation energy can be regarded as a size-intensive feature of a system, dynamic correlation is an extensive quantity with respect to the size of the system,

\footnotetext{
a) Author to whom correspondence should be addressed: francesco. aquilante@unibo.it

b) Current address: Departement' de Chimie Physique, Sciences II, 30, Quai E. Ansermet, 1211 Geneva, Switzerland.
}

and therefore its evaluation becomes very costly in large molecules. This is highlighted by the slow convergence shown by the dynamic correlation energy in terms of the amount of molecular orbitals (MOs), which gives rise to a sheer increase in the computational cost for quantitative analysis even for systems of small to medium size when using diffuse and accurate basis sets. Several cost-effective approximations have been proposed over the years to overcome this hurdle, being based on different approaches. Although the use of numerical grids as an alternative to standard basis sets is certainly a powerful means to avoid such slow convergence, it comes with its own shortcomings and remains a far less popular choice in quan-tum chemistry. ${ }^{4}$ At the opposite of the spectrum, one of the most promising approaches was pioneered by Pulay ${ }^{5}$ and oth-ers, 6,7 where localised natural orbitals (NOs) ${ }^{8}$ were employed to reduce the computational cost by truncating the virtual space and thus removing them from the correlation step. Owing to the fact that electron correlation is mostly of pairwise nature, the construction of optimized two-particle functions (gemi-nals) represents a route to tackle the same problem within the standard basis set framework. This is the rationale behind a number of approaches that have regained popularity over the last decade. These methods have nowadays regained popularity, being formulated through the pair natural orbital (PNO), local (LPNO), or domain-based (DLPNO) formulations, ${ }^{9-12}$ as well as through explicitly correlated ${ }^{13,14}$ and orbital-specific virtual (OSV) approaches. ${ }^{15,16}$ It is worth noting that PNO and OSV techniques rely on the removal of the virtual space from the correlation treatment in different ways, using PNOs and by considering a threshold on the "diagonal" amplitudes as different criteria, ${ }^{15,16}$ while explicitly correlated approaches rely exclusively on the construction of two-particle functions with orbital deletion. PNO-based, OSV-based, and explicitly based correlation methods provide a robust means to improve upon the problem of slow convergence of the correlation energy vs. basis set completeness but their implementation into effi-cient algorithms for the most advanced electron correlation 
methods is a serious endeavour. Despite their outstanding success in reducing the scaling of the dynamic correlation energy evaluation, these so-called local correlation methods $9,10,15-17$ are not free from various shortcomings. 18

A practical workaround is then to renounce describing the pairwise nature of the correlation energy and adopt a single-particle picture that is specifically designed to include correlation effects from the virtual space. This idea, pioneered by Barr and Davidson in the seventies, ${ }^{19}$ has been recurrently used over the years and implemented in a series of many-body perturbation theory approaches. ${ }^{20-26}$ In this context, and particularly for the methodology used in the present work, computational costs are ultimately reduced in practical computations by showing that it is possible to retain only a subset of such one-particle states that effectively spans the unoccupied (virtual) space, in the sense of account-ing for the overwhelming majority of the dynamic electron correlation.

\section{THEORY}

One possibility for low-cost formulations of many-body theory in quantum chemistry arises from the fact that dynamic correlation effects produce deviations from the mean-field electron density that are of much smaller entity than those produced by static correlations, and thus can be regarded as small perturbations. Drawing on this observation and a num-ber of similar insights into the problem of compacting the virtual orbital space, ${ }^{20-26}$ we have shown that the use of oppor-tunely defined approximate natural orbitals (NOs) allows such type of truncation with negligible and controllable errors in the computed energies and properties. ${ }^{27,28}$ The idea is to use the following density-like matrix:

$$
\dot{\nu}_{a b}^{(2)}={ }_{i c} t_{i i}^{a c} t_{i i}^{c b},
$$

where the employed approximate amplitudes are given by

$$
t i j^{a b}=\frac{(a i j b j)}{a i+b j j} .
$$

The indices $a, b,:::$ refer to the virtual orbitals, whereas

$i, j,::$ : correspond to orbitals that are either doubly occupied or partially occupied but with negative energy. The two-electron integrals (ailbj) present in Eq. (2) are conveniently com-puted through Cholesky decomposition-based density fitting (DF) 29,30 and the orbital energies 's result from diagonaliz-ing the corresponding generalized Fock matrix. The latter is defined $^{31}$ for the entire MO space as

$$
F_{p q}=h_{p q}+_{r s} U_{r s(p q j r s)} \quad \overline{2} \text { (psiqr) }
$$

with Drs indicating the one-particle density matrix elements of the reference wave function (CASSCF or HF) and $h p q$ referring to the usual one-electron integrals. Among the many possible definitions, ${ }^{32,33}$ this definition is such that the diagonal ele-ments with inactive and external orbital index of the Fock matrix always correspond to orbital energies in the sense of Koopmans' theorem.
The matrix of Eq. (1) is symmetric positive definite, and for large basis sets, it shows the typical clustering of its eigenvalues towards zero. This means that very few NOs defined through its diagonalization have a significant occupation, as represented by the corresponding eigenvalue. In other words, the matrix of Eq. (1) is a quantity that can be used to spot the onset of linear dependences in the virtual orbital space. Diagonalization of $D^{(2)}$ provides a new set of orthonormal (natural) orbitals that can be used in higher-level correlated treatments. As the sequence of eigenvalues of these NOs can be ordered, a systematic truncation of the virtual space is possible. Although only a part of the total correlation energy is recovered upon truncation, this approach has been shown to be extremely effective in reproducing relative energies for multiconfigurational second-order perturbation theory (CASPT2) ${ }^{27,28}$ and goes by the name of Frozen Natural Orbital (FNO) approach. In particular, FNO can produce a smooth potential energy surface (PES) if the fraction of NOs to be retained is determined based on the following quantity:

$$
()=\frac{\mathbf{P}^{a a}}{\operatorname{Tr}\left(D^{(2)}\right)},
$$

where the first largest eigenvalues and the trace of the matrix defined in Eq. (1) are used in the above formula. For a fixed value of , the number of retained virtual NOs is dynam-ically determined along a PES scan. Such quantity is obviously nonnegative and bound from above by the value $=1$-as it is proportional to the number of retained NOs - and represents a measure of the amount of electronic charge that will populate the virtual space as result of the many-body correlation treatment. At the same time, the amount of dynamic correlation energy recovered is a monotonically increasing function $F$ of

such that the whole dynamic correlation energy is attained as $E_{C}=F(1)$.

In this paper, we show that although the function $F()$ is unknown and non-universal, by means of a convenient representation in Taylor series, the value of $E_{C}$ within a given $a b$ initio method can be approximated with great accuracy by the application of the so-called Shanks transformation 34 for non-linear series convergence acceleration. In fact, assuming $F($ ) is differentiable, up to a 2nd-order Taylor expansion, we have

$$
E_{C}=F(1) \quad F()+F^{0}()(1)+\frac{1}{2} F^{00}()(1 \quad)^{2} .
$$

The above expression can be matched to the first three terms of an infinite series that converges to the total correlation energy

$$
E_{C}=0+1+2+.
$$

The first term, $0=F($ ), is obviously negative by defini-tion of correlation energy. Similarly, $1=F^{0}()(1)$ is also negative due to the monotonic behavior of $F()$, whereas $2=2^{1} F^{00}()(1)^{2}$ is positive for sufficiently close to 1 , as expected in a basis of approximate NOs. The partial sums $A_{0}=0, A_{1}=0+1$, and $A_{2}$ $=0+1+2$ thus show an oscil-latory behavior that suggests the use of the Shanks method ${ }^{34}$ to accelerate the convergence of the series of Eq. (6). Shanks transformation is defined as follows: given the input sequence 
of partial sums ( $\left.A_{0}, A_{1}, A_{2},:::\right)$, the transformed sequence is constructed as

$$
S\left(A_{k}\right)=\begin{array}{llll}
A_{k+1} A_{k} & 1 & A_{k}^{2} \\
A_{k+1}+A_{k} & & 2 A_{k}
\end{array} .
$$

The Shanks transformation gives the exact limit in a single step if the original sequence represents a geometric series. For arbitrary sequences, applying Shanks transformation often improves the convergence substantially, and this is espe-cially true for alternating series. Furthermore, Shanks trans-formation can be applied iteratively to produce $S\left(S\left(A_{k}\right)\right), S\left(S\left(S\left(A_{k}\right)\right)\right)$, : $:$ : by means of successive applications of Eq.

(7). Noticeably, if one stops at the first Shanks iterate, the form of the approximation is also analogous to Aitken's delta-squared process, ${ }^{35}$ with the difference that Shanks operates on the partial sums rather than operating on the terms of the series.

Despite its excellent performance as a series acceleration method, the Shanks transformation does suffer from undeniably unpleasant complications. In the language of mathematics, one would refer to the Shanks transformation as being neither linear nor regular. ${ }^{35}$ Nonlinearity and nonreg-ularity are essential ingredients of any powerful method for sequence transformation, despite giving rise to undesired con-sequences when applied to problems in physical sciences. In our case, nonlinearity translates into lack of size-consistency of the extrapolated energy. At the same time, size-extensivity intended as the correct scaling of the extrapolated energy with the number of electrons is guaranteed by the mathemat-ical property of these types of series extrapolation methods known as translational invariance. ${ }^{35}$ In earlier formulations of FNO, ${ }^{25,26,28}$ potential energy surfaces were not guaranteed to be smooth, and this was partly a result of the loss of sizeconsistency of the original quantum chemical method upon truncation based solely on the fraction of NOs retained. This problem has been addressed by the present authors ${ }^{27}$ via the introduction of the metric of Eq. (4), through which it is possible to control the amount of correlation energy retained at every step of a fragmentation process. Hence, this more recent type of FNO truncation preserves the properties of size-consistency of the quantum chemical method used to an extend that is practically indistinguishable from a truly size-consistent theory. Therefore, we argue in favor of the fact that a similar behavior is to be expected when using Shanks estimated based on the current FNO implementation, despite the nonlinear (nonstrictly size-consistent) nature of the series extrapolation technique.

The main result that we report here is to demonstrate by numerical examples that even the first Shanks extrapola-tion alone, $S\left(A_{1}\right)$ from Eqs. (5)-(7), computed by means of three different FNO approximations to the correlation energy invariably provides an improved estimate of the reference cor-

relation energy $E_{C}$. Applying the Shanks requires only FNO calculations that are much cheaper than the full-MO calculation, and therefore, this straightforward result supplies a computational shortcut to account for the total correlation energy within a given $a b$ initio method at a fraction of its cost. In order to compute the three terms of Eq. (5), we need to estimate the first and second derivatives of the unknown function $F($ ). This is achieved by means of the finite difference approximation.

\section{RESULTS AND DISCUSSION}

We now present a series of numerical results starting with the assessment of the usefulness of this type of Shanks extrapolation on vertical excitation energies computed using the FNO-CASPT2 method, ${ }^{27}$ employing an in-house development version of the MOLCAS 8 package. ${ }^{36}$ As the choice of which truncations for the numerical differentiation should be used is somewhat arbitrary, we investigated how triplets of FNO calculations can be combined to obtain energy estimates that are invariably closer to the full-MO Cholesky decompo-sition (CD)-CASPT2 value both in terms of total and relative energies. Thymine is used as an example given its biological relevance 37 and the fact that it features lowlying and no states, requiring different amounts of dynamic correlation for their proper description. ${ }^{38}$

Figure 1 shows how monotonically approaches the fullMO CD-CASPT2 solution $(=1)$, large enough values $(=0.97$ 0.99 ) being sufficient within the FNO framework to account for the majority of the dynamic correlation. It also provides valuable insights into its dependence on the basis set size: larger basis sets are optimal for FNO truncation as the virtual orbital space can be efficiently mapped onto a much more compact set of NOs, thus producing significant speed-ups. ${ }^{27}$ These FNO results provide remarkably accurate vertical excitation energies: values within $0.2 \mathrm{eV}$ as compared to CDCASPT2 estimates are obtained when considering $>0.97$, whereas values within a tenth of an $\mathrm{eV}$ can be expected for

$>0.99$, the values being in agreement with reports from the literature on highly correlated calculations. ${ }^{39-41}$ Figure 1(d) shows a zoom-in view displaying the most correlated FNO treatments $(>0.9)$, and how these may be combined to extrapolate the total energy employing the Shanks procedure. As can be seen, by employing three highly correlated FNO energies, namely, at $=98.5 \%, 99 \%$, and $99.5 \%$, an extrap-olated value even closer to the CDCASPT2 solution can be attained. It is worth noting that regardless of the adequate accu-racy of the most correlated approach $(=0.995)$, the Shanks extrapolation is capable of further improving the estimate by combining it with less expensive and correlated compu-tations that may be carried out simultaneously owing to trivial parallelization.

An in-depth look at the Shanks extrapolation for computing vertical excitation energies is given in Fig. 2, where three different triplets of points are displayed together with their deviation from the CD-CASPT2 reference. One impor-tant aspect to note is that in almost all cases, the extrapo-lated energy obtained is closer to the reference value than the most correlated point employed in the Shanks. The least correlated Shanks series uses FNO-(96\%, 97\%, and 98\%), providing remarkable results at the ANO-L-VDZP and ANO-L-VQZP basis sets leading to errors less than a tenth of an $\mathrm{eV}$ from the CD-CASPT2 reference. Larger errors are observed for ANO-LVTZP basis set, likely originating in an unbal-anced description of the ground state compared to the excited states. However, starting with the triplet FNO-(97\%, 98\%, 

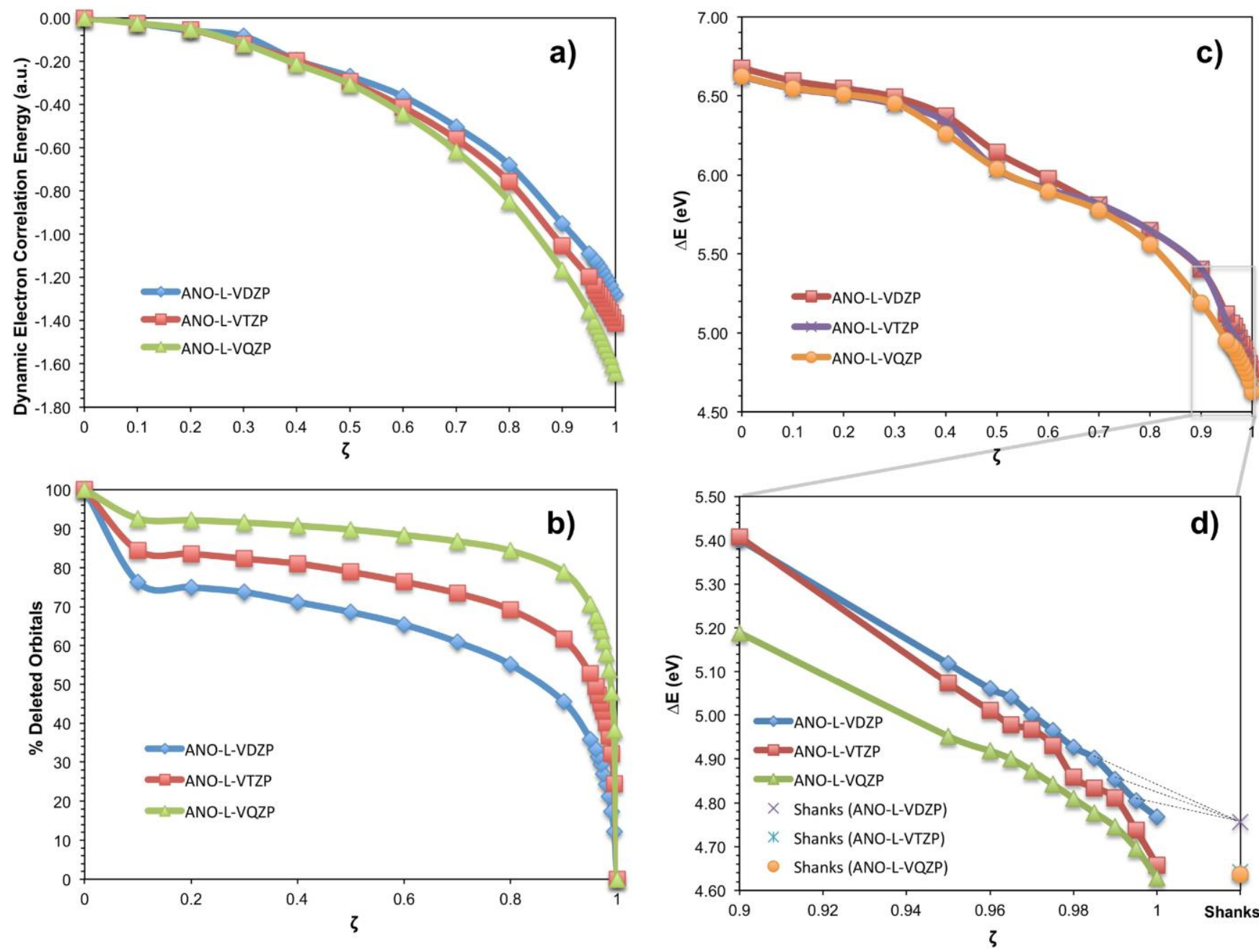

FIG. 1. FNO and Shanks performance with different basis sets for the state of thymine: (a) dynamic electron correlation with different basis set of the ground state, (b) percentage of deleted orbitals in the different FNO computations, (c) vertical excitation energies at different basis sets, and (d) the zoom-in view of the highly correlated FNO computations and comparison with the Shanks estimates. All FNO computations are displayed with respect to the correlation retained as expressed by. Dashed lines represent the points employed for the Shanks-extrapolated values shown, referring to an extrapolation employing $98.5 \%, 99 \%$, and $99.5 \%$ of the trace.

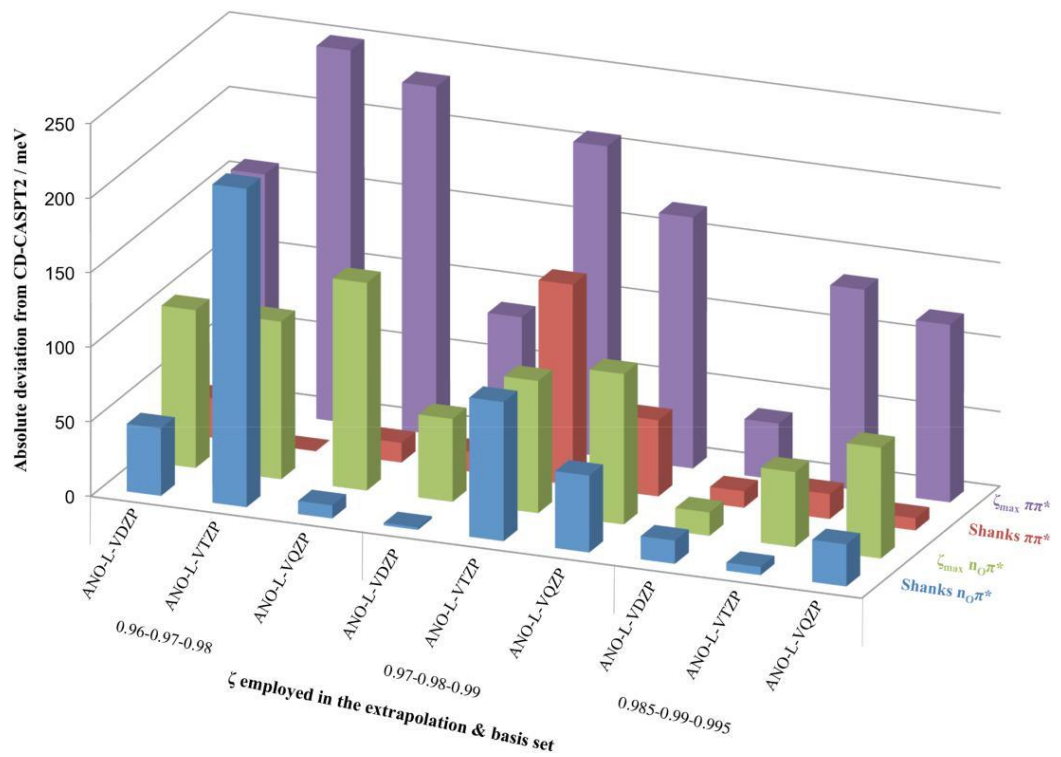

FIG. 2. FNO and Shanks-extrapolated absolute deviations in vertical excitation energies for the lowestlying electronic states of thymine as compared to the CD-CASPT2 reference results. 
and $99 \%$ ), the worsening of the results upon extrapolation disappears also for the ANO-L-VTZP basis set, yielding a satisfactory improvement for the estimated excitation energies across the different basis sets and type of excited state. Furthermore, accuracy improves when shifting the triplet towards larger max, as shown by the FNO-(98.5\%, 99\%, and 99.5\%) triplet which leads to errors within a hundredth of an $\mathrm{eV}$ with respect to the reference. All sets of triplets perform better in conjunction with large basis sets, and the accuracy attained seems to be consistent across the different states of diverse nature analyzed (i.e., for covalent no and ionic states). ${ }^{27}$ The Shanks extrapolation appears therefore as an encourag-ing option to push the calculation of excitation energies by means of correlation methods towards the basis set limit in a more affordable manner. To this end, only a more thor-ough benchmarking could ultimately provide an answer to the question of which is the cheapest and yet general triplet of points for the extrapolation. At this stage, we aimed at a proof of concept of possible uses and applications of the this particular type of Shanks extrapolation; hence, the idea has been assessed only with respect to the need to obtain a triplet of points that would always give the highest accuracy, and there-fore we may have overlooked solutions with better trade-off between accuracy and costs. The results of the current investi-gation indicate that the choice of FNO-(97\%, $98 \%$, and $99 \%$ ) is the minimal triple of points that ensures a very high accuracy of the proposed Shanks extrapolation in computing excitation energies.

Despite having showcased it here so far for CASPT2, the FNO method was actually introduced in many-body perturbation theory single-reference methods, ${ }^{21,25,26}$ where the higher computational scaling of the methods justify even more the interest in its use. Our algorithm for FNO selection ${ }^{27}$ has therefore been adapted for use in conjunction with MP2, coupledcluster single and double (CCSD), and coupled-cluster sin-gle and doubles with perturbative triple $[\mathrm{CCSD}(\mathrm{T})]$ methods and here assessed together with the Shanks extrapolation to explore its potential for the study of ground state reactivity
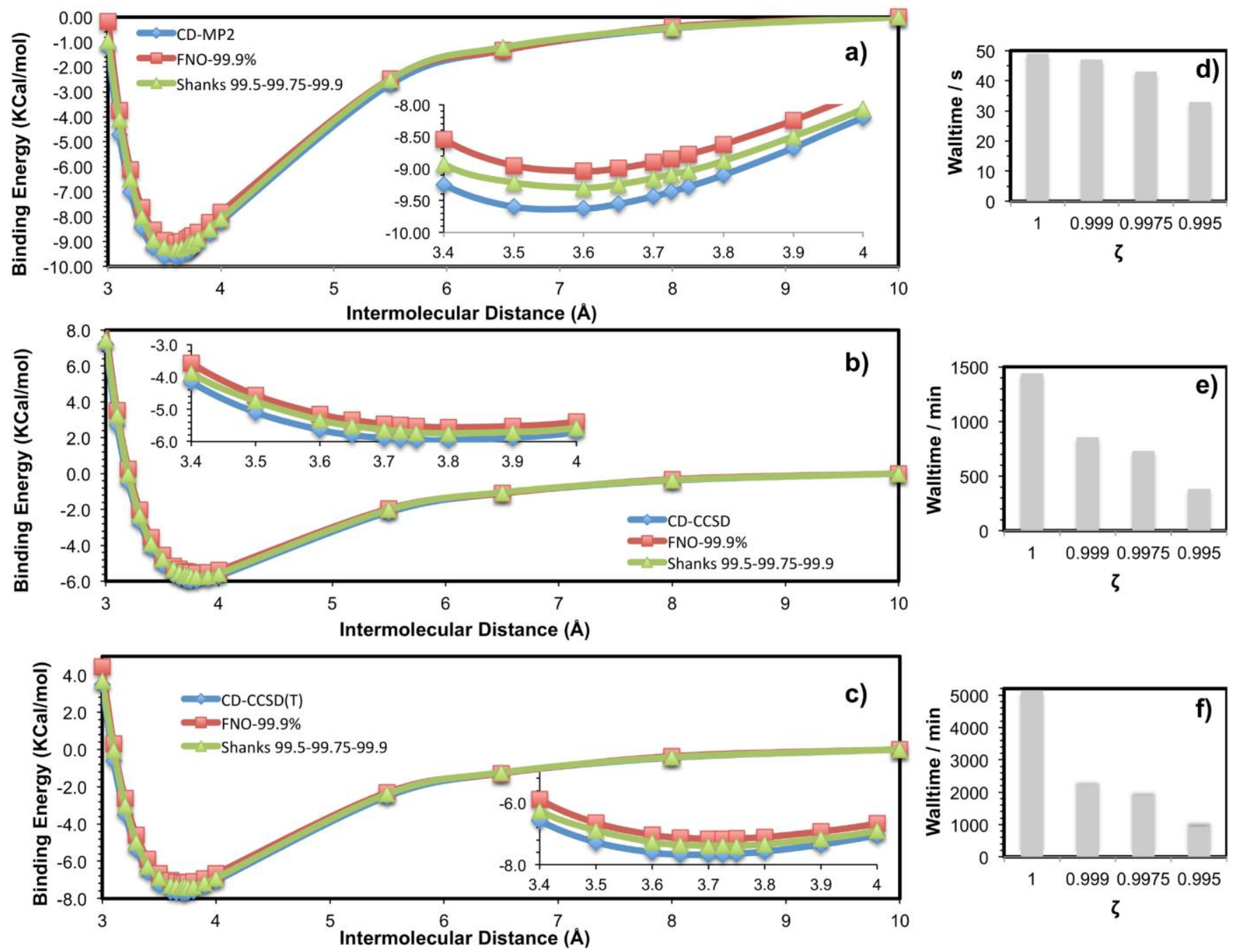

FIG. 3. Potential energy surface of the fully stacked face-to-face benzene-benzene dimer at the MP2 (a), CCSD (b), and CCSD(T) (c) employing an ANO-LVTZP basis set and CD-based algorithms. Results from each method is displayed against a highly correlated FNO ( $=0.999)$ as well as with values obtained from the Shanks extrapolation for FNO-(99.5\%, 99.75\%, and 99.9\%). (d)-(f) show the relative speed-ups attained for the different values with respect to the full-MO Cholesky decomposition solution $(=1)$ for MP2, CCSD, and CCSD(T), respectively. A zoom-in view of the 3.4-4 $\AA E$ region is also provided in each respective panel. 
TABLE I. MP2, CCSD, and CCSD(T) FNO and Shanks extrapolated estimates for the binding energy of the fully face-to-face stacked benzene-benzene dimer. The full-MO Cholesky Decomposition (CD) results are shown for comparison. Binding energies ( $E$, in $\mathrm{kcal} / \mathrm{mol})$ and intermolecular distances $\left(R_{0}\right.$, in $\AA$ ) refer to those obtained at the minima of the potential energy curves depicted in Fig. 3.

\begin{tabular}{|c|c|c|c|c|c|c|c|c|c|}
\hline & \multicolumn{3}{|c|}{ MP2 } & \multicolumn{3}{|c|}{ CCSD } & \multicolumn{3}{|c|}{$\operatorname{CCSD}(\mathrm{T})$} \\
\hline & $\mathrm{CD}$ & FNO- $99.9 \%$ & Shanks & $\mathrm{CD}$ & FNO-99.9\% & Shanks & $\mathrm{CD}$ & FNO- $99.9 \%$ & Shanks \\
\hline$E$ & 9.624 & 9.040 & 9.298 & 5.963 & 5.562 & 5.745 & 7.681 & 7.163 & 7.384 \\
\hline$R_{0}$ & 3.8 & 3.8 & 3.8 & 3.6 & 3.6 & 3.6 & 3.7 & 3.7 & 3.7 \\
\hline
\end{tabular}

with highly correlated methods. The fully stacked face-to-face benzene dimer and its potential energy surface along the stacking distance are chosen as the case-study example due to its importance as a representative of aromatic non-covalent interactions. ${ }^{42,43}$ The results reported here are obtained without employing corrections for basis set superposition errors, as the numerical demonstration given is aimed at providing a proof of principle rather than an accurate measure of the binding energy of benzene, which has been reported elsewhere. ${ }^{44-47}$ Neverthe-less, the ANO-L-VTZP basis set employed is expected to be large enough for these corrections to be of little impact. This is confirmed by the fact that the values obtained appear to be in very good agreement with the literature data.

Figure 3 displays the potential energy surfaces of the benzene-benzene stacked dimer at the MP2, CCSD, and CCSD(T) levels of theory. As it can be seen, qualitatively similar profiles are obtained being quantitatively vastly different, particularly in the case of MP2 where an overestimation of the binding energy is observed. It is worth noting that our FNO implementation is capable of reproducing accurately the energy along the full potential energy curve, showcasing how the orbital removal criterion is solid enough to properly repre-sent the energy in different geometries. The highly correlated FNO approach used $(=0.999)$ is capable of recovering most of the dynamic correlation in each of the different methods, yielding errors of around $0.5 \mathrm{kcal} / \mathrm{mol}$ in the binding energy with respect to the reference and placing the minimum at the same intermolecular distance (see Table I). Correspond-ingly, the speed-ups are significant, as s20\% of the virtual space is removed even in this case. At no additional costs, Shanks extrapolated values manage to improve on the FNO estimates, placing the binding energy to within $0.3 \mathrm{kcal} / \mathrm{mol}$ from the reference. Despite being a small gain in absolute terms in this particular case-although it still represents a nearly 50\% reduction in error-the fact that the Shanks extrap-olation outperforms the highly correlated FNO shows how unequivocally useful this idea is. The possibility to achieve systematically closer energies with respect to the fully correlated solution thus opens the way for reproducing potential energy surfaces within chemical accuracy of the reference at substantially reduced computational costs. It is also worth noting that both FNO and Shanks extrapolated curves show a smooth character analogous to the reference results, ${ }^{48,49}$ unlike previous natural orbital-based or localized formulations, ${ }^{18}$ while not requiring any predefined orbital domain selection or additional input parameters. ${ }^{50}$ This is an indication of the valid-ity of the Shanks, as well as of our latest FNO protocol, ${ }^{27}$ as methods for general-purpose chemical reactivity studies. Finally, we note that at variance with the case of CD-CASPT2 vertical excitation energies, where a FNO-(97\%, 98\%, and 99\%) triple suffices for accurate results, the Shanks extrapolation requires higher correlated FNO calculations $(99.5 \%$,

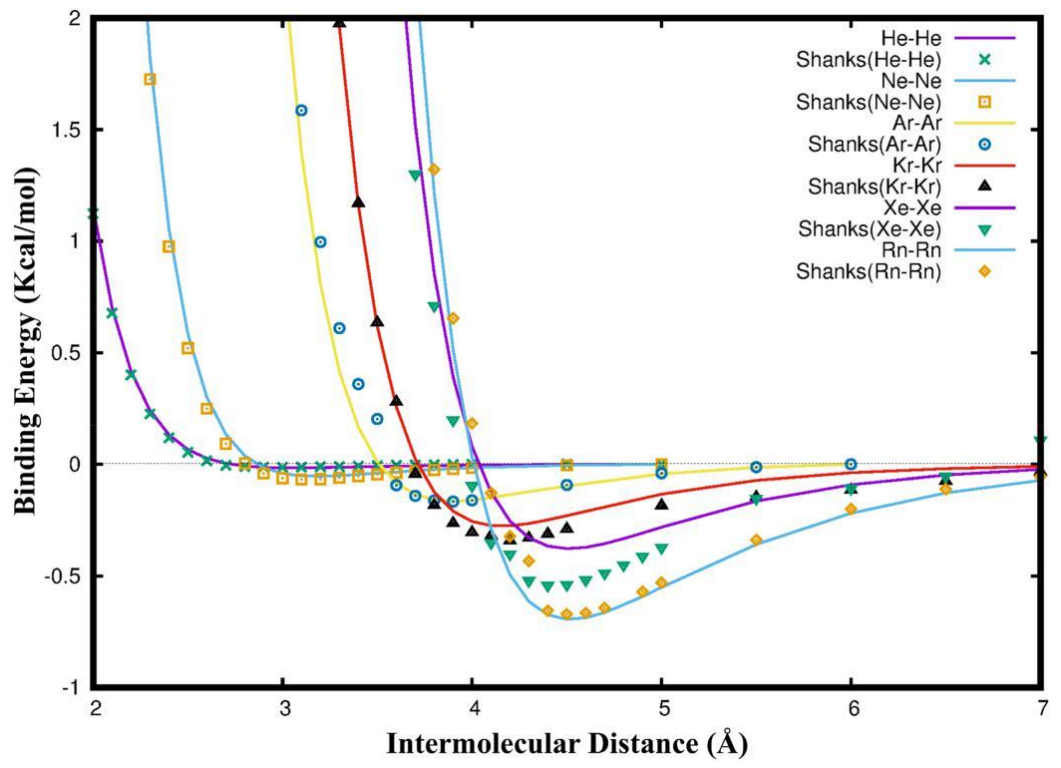

FIG. 4. Potential energy surfaces of the diatomic rare gas dimers at the CCSD(T) level of theory employ-ing an ANO-RCC-VQZP basis set and CD-based algorithms. $\operatorname{CCSD}(\mathrm{T})$ results are displayed against Shanksextrapolated estimates employing FNO- $(99.7 \%, 99.8 \%$, and $99.99 \%$ ). 

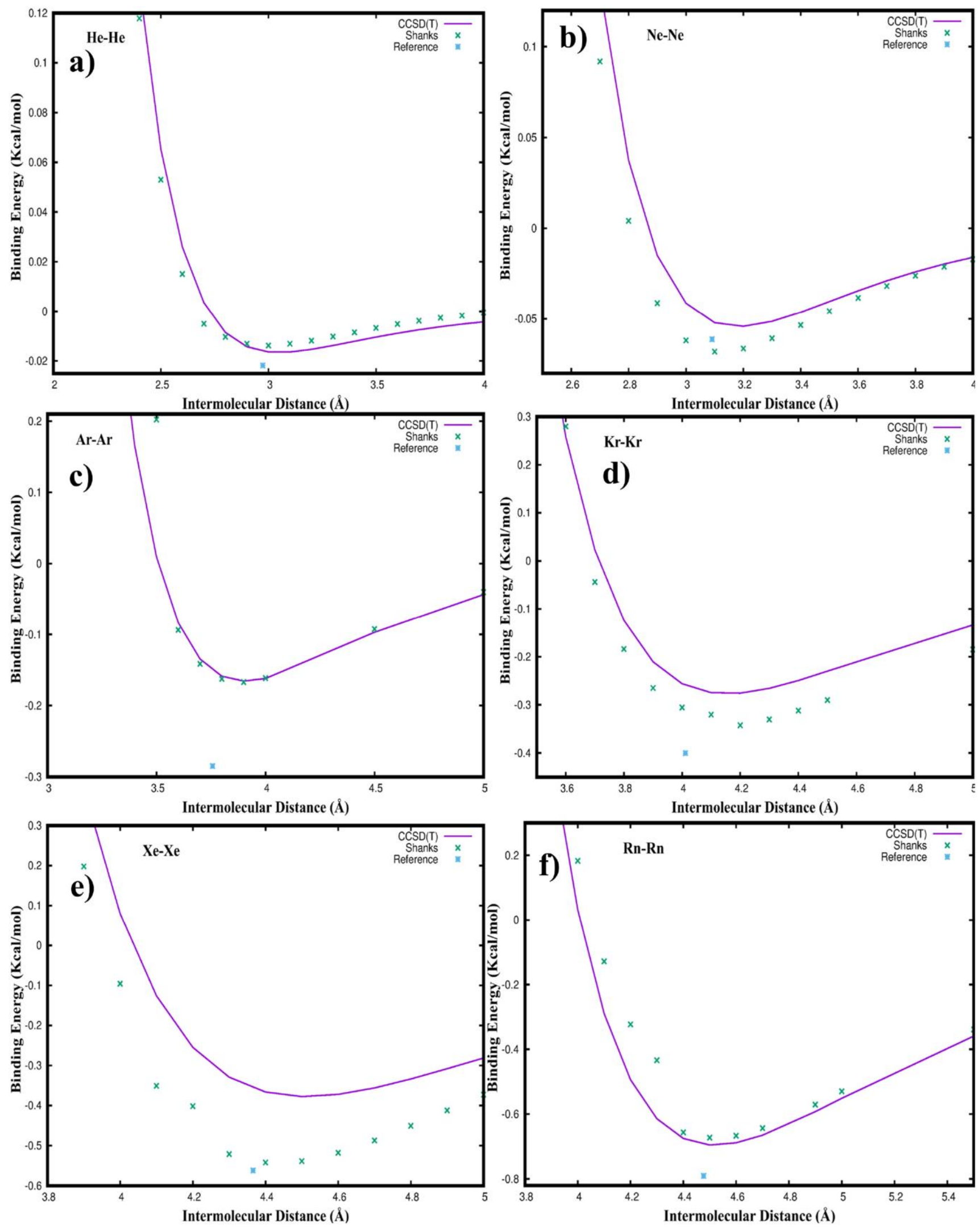

FIG. 5. Potential energy surfaces of the diatomic rare gas dimers at the CCSD(T) and Shanks FNO-(99.7\%, 99.8\%, and 99.99\%) levels of theory employing an ANORCC-VQZP basis set and CD-based algorithms together with high-level reference values reported by Tang and Toennies. ${ }^{2}$ The different curves for (a) He-He, (b) Ne$\mathrm{Ne}$, (c) $\mathrm{Ar}-\mathrm{Ar}$, (d) $\mathrm{Kr}-\mathrm{Kr}$, (e) $\mathrm{Xe}-\mathrm{Xe}$, and (f) $\mathrm{Rn}-\mathrm{Rn}$ are shown, displaying the most relevant intermolecular distances near the equilibrium.

99.75\%, 99.9\%) when aimed at reproducing potential energy surfaces with coupled cluster theory. Nonetheless, this is still a favourable situation from the computational costs' point of view, especially for large basis sets where even at such 
high values of , there is significant removal of the virtual orbitals.

As shown above for the benzene dimer, tighter thresh-olds (more correlated FNO computations) are required to obtain correct Shanks-extrapolated values in agreement with the $\mathrm{CCSD}(\mathrm{T})$ reference as compared to those used for obtain-ing vertical excitation energies with CASPT2. This is partially due to the very different properties considered: on the one hand, vertical excitation energies display values that range several $\mathrm{eV}$ in magnitude and where small deviations in the total energies employed can be disregarded as negligible errors and sometimes recovered due to error cancellation as we con-sider pairs of electronic states. On the other hand, as shown in the benzene dimer example, ground state binding energies are embodied by much smaller quantities, in the order of few $\mathrm{Kcal} / \mathrm{mol}$, which rely on much more accurate estimates of the total energy and will thus require tighter thresholds and more correlated approaches for their correct description.

To showcase this, we consider next the potential energy surfaces of the diatomic rare gas homo-dimers ( $\mathrm{He}-\mathrm{He}, \mathrm{Ne}-$ $\mathrm{Ne}, \mathrm{Ar}-\mathrm{Ar}, \mathrm{Kr}-\mathrm{Kr}, \mathrm{Xe}-\mathrm{Xe}$, and $\mathrm{Rn}-\mathrm{Rn}$ ) at the $\mathrm{CCSD}(\mathrm{T})$ level and a range of Shanks extrapolated schemes computed mak-ing use of the ANO-RCC-VQZP basis set. ${ }^{51}$ Figure 4 shows the overall potential energy curves of the different dimers and their energy profiles. As can be seen, Shanks estimates are overall able to reproduce almost quantitatively for all cases the potential energy surfaces of these systems, displaying a smooth behaviour and, with the exception of $\mathrm{Xe}-\mathrm{Xe}$, being within $\mathrm{s} 0.01 \mathrm{Kcal} / \mathrm{mol}$ of the full $\mathrm{CCSD}(\mathrm{T})$ computation.

A more in-depth discussion is given in Fig. 5, where a zoomin view of the different diatomics around their equilib-rium distances are given and compared to the accurate refer-ence values summarised in the work of Tang and Toennies. ${ }^{52}$ As can be seen in Fig. 5(a), He-He presents a very shallow min-imum at s0.02 $\mathrm{Kcal} / \mathrm{mol}$ according to our $\operatorname{CCSD}(\mathrm{T})$ estimates, close to the exact quantum Monte Carlo values of Anderson, ${ }^{53}$ located at a slightly displaced s $0.05 \AA$, our Shanks estimates closely replicating the full $\operatorname{CCSD}(\mathrm{T})$ data. It can therefore be considered an extremely challenging and worth studying case for our Shanks extrapolation method, as errors in the order of

$10^{5}$ hartree already show discrepancies with respect to the reference. Figure 5(b) shows the potential energy curve of $\mathrm{Ne}-\mathrm{Ne}$ with reference values taken from the works of Aziz and Slaman. ${ }^{54}$ Our CCSD(T) data show an almost quantitative agreement with reference data, Shanks extrapolated values being slightly overestimated and overshooting the binding energy at the minimum by $\mathrm{s} 0.01 \mathrm{Kcal} / \mathrm{mol}$ while displaying its mini-mum at the right intermolecular distance. The Ar-Ar surface is given in Fig. 5(c) and shows the largest discrepancies between

the reference, based on a modified potential developed by Aziz, ${ }^{55}$ and the computed data at both $\operatorname{CCSD}(T)$ and the extrap-olated Shanks values. As can be seen, excellent agreement is found between the extrapolated Shanks and the CCSD(T) values except at short distances (s3.5 $\mathrm{A}$ ), these being over $0.1 \mathrm{Kcal} / \mathrm{mol}$ underestimated with respect to the reference value while featuring the minimum at roughly the same intermolecular distance. Figure 5(d) displays $\mathrm{Kr}-\mathrm{Kr}$, together with the reference value obtained by Dham et al. based on a modified potential. ${ }^{56}$ The Shanks extrapolated values overestimate the $\operatorname{CCSD}(\mathrm{T})$ values, and both approaches underestimate the reference binding energy while overestimating the intermolecular equilibrium distance. $\mathrm{Xe}-\mathrm{Xe}$ is shown in Fig. 5(e) and, similar to $\mathrm{Kr}-\mathrm{Kr}$, displays Shanks-extrapolated values slightly overestimated with respect to $\operatorname{CCSD}(\mathrm{T})$. Upon comparison with the reference values of Dham et al., ${ }^{57}$ our $\operatorname{CCSD}(\mathrm{T})$ values appear to underestimate the binding energy and to be characterised by a somewhat larger equilibrium distance. Shanks estimates show agreement with the reference despite being due to their overestimated nature. Finally, Fig. 5(f) shows the curves for $\mathrm{Rn}-\mathrm{Rn}$, compared with reference values of Runeberg and Pyykko“ obtained with pseudopotentials. ${ }^{58}$ Shanks extrapolated values appear to display larger errors at shorter intermolecular distances with respect to $\operatorname{CCSD}(\mathrm{T})$ while yield-ing accurate energies around the equilibrium geometry. Both Shanks and $\operatorname{CCSD}(\mathrm{T})$ place the minimum close to the reference value at $\mathrm{s} 4.48 \AA$, while being underestimated in around $0.1 \mathrm{Kcal} / \mathrm{mol}$.

Overall, qualitative and sometimes even quantitative agreement is reached by the Shanks extrapolated values with respect to $\operatorname{CCSD}(\mathrm{T})$ for all rare gas homo-dimers. Upon comparison with reference values, it can be seen how $\operatorname{CCSD}(\mathrm{T})$ appears to overestimate slightly the binding energies so obtained while providing very similar equilibrium distances. The Shanks extrapolated values are shown to generally lie on top of the parent $\operatorname{CCSD}(\mathrm{T})$ computation while slightly overestimating binding energy on some cases. It is worth noting that highly correlated FNO computations were employed in the extrapolation, being used as a proof-of-concept and potentially being improvable by using less correlated and computationally expensive points in favour of a more cost effective approach. More importantly, however, the profiles so obtained by the Shanks extrapolated values appear to be rather smooth and thus validate this approach even for extremely challenging cases such as rare gas dimers, where binding energies of less than 1 $\mathrm{Kcal} / \mathrm{mol}$ are expected. These errors, which are below the intrinsic expected error of methods such as $\operatorname{CCSD}(\mathrm{T})$, are therefore considered affordable and validate the potential use of the Shanks extrapolation for large scale applications where big molecular systems and extensive basis sets are employed.

\section{SUMMARY}

In conclusion, we have shown by numerical investiga-tions how a specific form of series extrapolation (the Shanks transformation) can be used as a workaround to the slow convergence of the correlation energy calculation with respect to the size of the one-particle basis in a many-body treatment. With a suitable choice of pre-ordered approximate natural orbitals, three calculations in a partially correlated orbital space are performed and the resulting energies are mapped onto an infinite series converging to the correlation energy for the full orbital space calculation. The Shanks transformation is then used to improve the convergence of the truncated series towards its infinite sum, hence towards the expected result for the full many-body correlation treatment. The method has been shown to be applicable to ground and excited state prob-lems and is expected to be trivially extended to other known 
natural orbital-based or local correlation methods. Deviations in the predicted energetics are one order of magnitude smaller than the intrinsic error of the given many-body correlation treatment even for the most difficult cases. Coupled with the resulting ease of computation compared to the full calcula-tion, this fact indicates that the proposed idea is an effective means to overcome the one-particle scaling wall of many-body quantum chemistry calculations.

\section{ACKNOWLEDGMENTS}

F.A. acknowledges financial support from Italian Ministry of Education and Research (MIUR) - Grant No. RBFR1248UI. J.S.-M. acknowledges Project No. CTQ-2014-58624-P of the Spanish MINECO (Ministerio de Economia y Competitividad). M.G. acknowledges support by the European Research Council Advanced Grant STRATUS (ERC-2011-AdG No. 291198) and of the French Agence National de la Recherche (FEMTO2DNA, No. ANR-15-CE29-0010).

${ }^{1}$ B. O. Roos, in Advances in Chemical Physics; Ab Initio Methods in Quantum Chemistry-II, edited by K. P. Lawley (John Wiley and Sons, Inc., 1987), Vol. 69, pp. 399-445.

2F. Aquilante, P.-Å. Malmqvist, T. B. Pedersen, A. Ghosh, and B. O. Roos, J. Chem Theory Comput. 4, 694 (2008).

3F. Aquilante, T. B. Pedersen, R. Lindh, B. O. Roos, A. S. de Meras,' and H. Koch, J. Chem. Phys. 129, 024113 (2008).

4S. Salomonson, I. Lindgren, and A. Martenson, Phys. Scr. 21, 351 (1980).

5 P. Pulay, Chem. Phys. Lett. 100, 151 (1983).

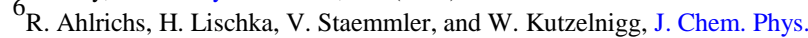
62, 1225 (1975).

${ }^{7}$ W. Meyer, J. Chem. Phys. 58, 1017 (1973).

${ }^{8}$ P. O. Lowdin," Int. J. Quantum Chem., Symp. 4, 231 (1971).

${ }^{9}$ M. Sparta and F. Neese, Chem. Soc. Rev. 43, 5032 (2014).

10D. G. Liakos, M. Sparta, M. K. Kesharwani, J. M. L. Martin, and F. Neese, J. Chem.

Theory Comput. 11, 1525 (2015).

11F. Neese, F. Wennmohs, and A. Hansen, J. Chem. Phys. 130, 114108 (2009).

12A. Hansen, D. G. Liakos, and F. Neese, J. Chem. Phys. 135, 214102 (2011).

13F. R. Manby, H.-J. Werner, T. B. Adler, and A. J. May, J. Chem. Phys. 124, 094103 (2006).

14W. Klopper, F. R. Manby, S. Ten-No, and E. F. Valeev, Int. Rev. Phys. Chem. 25, 427 (2006).

15J. Yang, G. K.-L. Chan, F. R. Manby, M. Schutz," and H.-J. Werner, J. Chem. Phys. 136 , $144105(2012)$

16M. Schutz," J. Yang, G. K.-L. Chan, F. R. Manby, and H.-J. Werner, J. Chem. Phys. 138 $054109(2013)$

17Z. Rolik and M. Kallay,' J. Chem. Phys. 135, 104111 (2011).

18 N. J. Russ and T. D. Crawford, J. Chem. Phys. 121, 691 (2004).

19T. L. Barr and E. R. Davidson, Phys. Rev. A 1, 644 (1970)

20M. Piton ak,' P. Neogrady,' V. Kello," and M. Urban, Mol. Phys. 104, 2277 (2006).

21C. Sosa, J. Geertsen, G. W. Trucks, R. J. Bartlett, and J. A. Franz, Chem. Phys. Lett. 159 148 (1989)

22L. Adamowicz, R. J. Bartlett, and A. J. Sadlej, J. Chem. Phys. 88, 5749 (1988).

23L. Adamowicz and R. J. Bartlett, J. Chem. Phys. 86, 6314 (1987).
24H. Jørgen, A. Jensen, P. Jørgensen, H. Ågren, and J. Olsen, J. Chem. Phys. 88, 3834 (1988).

25A. G. Taube and R. J. Bartlett, J. Chem. Phys. 128, 164101 (2008)

26A. G. Taube and R. J. Bartlett, Collect. Czech. Chem. Commun. 70, 837 (2005)

27J. Segarra-Mart' 1, M. Garavelli, and F. Aquilante, J. Chem. Theory Comput. 11, 3772 (2015).

${ }^{28}$ F. Aquilante, T. K. Todorova, L. Gagliardi, T. B. Pedersen, and B. O. Roos, J. Chem. Phys. 131, 034113 (2009).

29F. Aquilante, M. G. Delcey, T. B. Pedersen, I. F. Galvan,' and R. Lindh, Mol. Phys. 115 2052-2064 (2017).

${ }^{30}$ F. Aquilante, L. Boman, J. Bostrom," H. Koch, R. Lindh, A. S. de Meras,' and T. B. Pedersen, Linear-Scaling Techniques in Computational Chemistry and Physics (Springer Netherlands, 2011), pp. 301-343.

31D. Roca-Sanjuan,' F. Aquilante, and R. Lindh, Wiley Interdiscip. Rev.: Comput. Mol. Sci. 2, 585 (2012), ISSN 1759-0884

32K. Hirao and K. Nakatsuji, J. Chem. Phys. 59, 1457 (1973).

${ }^{33}$ R. Carbo' and J. M. Riera, Lecture Notes in Chemistry (Springer Verlag, 1976), Vol. 5.

34D. Shanks, J. Math. Phys. 34, 1 (1955).

${ }^{35}$ K. E. Atkinson, An Introduction to Numerical Analysis (Wiley, 1989).

${ }^{36}$ F. Aquilante, J. Autschbach, R. K. Carlson, L. F. Chibotaru, M. G. Delcey,

L. De Vico, I. F. Galvan,' N. Ferre,' L. M. Frutos, L. Gagliardi et al., J. Comput. Chem. 37, 506 (2016), ISSN 1096-987X.

${ }^{37}$ A. Giussani, J. Segarra-Mart'1, D. Roca-Sanjuan,' and M. Merchan,' Excita-tion of Nucleobases From a Computational Perspective I: Reaction Paths (Springer International Publishing, 2015), pp. 57-97, ISBN 978-3-31913371-3.

38J. Segarra-Mart' 1, A. Frances'-Monerris, D. Roca-Sanjuan,' and M. Merchan,' Molecules 21, 1666 (2016), ISSN 1420-3049.

${ }^{39}$ A. Giussani, J. Segarra-Mart'1, A. Nenov, I. Rivalta, A. Tolomelli, S. Mukamel, and M. Garavelli, Theor. Chem. Acc. 135, 121 (2016), ISSN 1432-2234.

40P. G. Szalay, T. Watson, A. Perera, V. F. Lotrich, and R. J. Bartlett, J. Phys. Chem. A 116, 6702 (2012).

41 A. J. Pepino, J. Segarra-Mart'1, A. Nenov, R. Improta, and M. Garavelli, J. Phys. Chem. Lett. 8, 1777 (2017)

42 K. Muller"-Dethlefs and P. Hobza, Chem. Rev. 100, 143 (2000).

43 .

44D. A. Sirianni, L. A. Burns, and C. D. Sherrill, J. Chem. Theory Comput. 13, 86 (2017).

45M. O. Sinnokrot, E. F. Valeev, and C. D. Sherrill, J. Am. Chem. Soc. 124, 10887 (2002)

46P. Hobza, H. L. Selzle, and E. W. Schlag, J. Am. Chem. Soc. 116, 3500 (1994).

47J. Rezac, M. Dubecky, P. Jurecka, and P. Hobza, Phys. Chem. Chem. Phys. 17, 19268 (2015).

48J. E. Subotnik and M. Head-Gordon, J. Chem. Phys. 123, 064108 (2005).

49J. E. Subotnik, A. Sodt, and M. Head-Gordon, J. Chem. Phys. 128, 034103 (2008).

50R. A. Mata and H.-J. Werner, J. Chem. Phys. 125, 184110 (2006).

51B. O. Roos, R. Lindh, P.-Å. Malmqvist, V. Veryazov, and P.-O. Widmark, J. Phys. Chem. A 108, $2851(2004)$

52K. T. Tang and J. P. Toennies, J. Chem. Phys. 118, 4976 (2003).

53J. B. Anderson, J. Chem. Phys. 115, 4546 (2001)

54R. A. Aziz and M. Slaman, Chem. Phys. 130, 187 (1989), ISSN 0301-0104

55R. A. Aziz, J. Chem. Phys. 99, 4518 (1993).

56A. K. Dham, A. Allnatt, W. J. Meath, and R. A. Aziz, Mol. Phys. 67, 1291 (1989).

57 A. K. Dham, W. J. Meath, A. Allnatt, R. A. Aziz, and M. Slaman, Chem. Phys. 142, 173 (1990), ISSN 0301-0104

58N. Runeberg and P. Pyykko," Int. J. Quantum Chem. 66, 131 (1998), ISSN 1097-461X. 